\title{
Development of Anti-Wear/Antioxidant Additives for High Quality Metalworking Fluid from Waste Petroleum Products
}

\author{
Noura Al Mehbad ${ }^{1 *}$, A. M. A. Omar ${ }^{2}$ \\ ${ }^{1}$ Faculty of Science, Najran University, Najran, Kingdom of Saudi Arabia; ${ }^{2}$ Egyptian Petroleum Research Institute, Cairo, Egypt. \\ Email: ${ }^{*}$ dr.n.almehbad@hotmail.com
}

Received November $21^{\text {st }}$, 2013; revised December $20^{\text {th }}, 2013$; accepted January $10^{\text {th }}, 2014$

Copyright (C) 2014 Noura al Mehbad, A. M. A. Omar. This is an open access article distributed under the Creative Commons Attribution License, which permits unrestricted use, distribution, and reproduction in any medium, provided the original work is properly cited. In accordance of the Creative Commons Attribution License all Copyrights (C 2014 are reserved for SCIRP and the owner of the intellectual property Noura al Mehbad, A. M. A. Omar. All Copyright (C) 2014 are guarded by law and by SCIRP as a guardian.

\begin{abstract}
Surfactants are used for formulation metal working fluids. These fluids are applied to the interface between a cutting tool and the metal working piece to provide friction reduction. In the present paper, poly aromatic ammonium sulphonate and polyaromatic benzyl triethanolamsonium ethoxylate with 6 ethylene oxide units were synthesized and blended with coupling agent like dodecyl alcohol and oleic acid to produce cutting fluid and evaluate as antiwear. Values of surface tension of these additives were measured in oil phase and consequently CMC was determined for all additives and their mixtures in oil phase. The efficiency of these additives depends on their chemical structure and the presence of ether oxygen brings about the association of the surfactant with metal surface, hence very good wetting properties. The antiwear characteristic of surfactants increases with increasing polar linkages in the aromatic moieties forming chelated and coordinated layer. This is due to the increase in the number of sites to chemisorption on the metal surface. The films formed are highly condensed ring aromatic layered structures. These films provide lower wear and depend on the type of surfactants concentration and sliding speed. The surface of metal was analyzed by SEM/EDX techniques. These studies have led to much clear evidence of the intimate relationship between the chemical structure of the surfactants and their film forming. More confirmation for suggested mechanism was investigated by measuring the area occupied per molecule of the surfactant in oil phase. The results indicate that the sulphonate group is less efficient than ethoxylate group. At the same time, the micelle of surfactants acts as oil dispersant and improves adsorption of oil on the metal surface.
\end{abstract}

\section{KEYWORDS}

Lube Oil; Antioxidants; Antiwears; Sulphonates; Ethoxylates; Phase Transfere Catalysts

\section{Introduction}

Metalworking can be defined as processes of modification of the shape of a metal piece. In all the metalworking processes, a considerable amount of heat is evolved due to the internal friction and the friction between tool and metal. Straight oils are used as rolling fluids for rolling of thin sheets of ferrous and nonferrous metals. Straight oils contain base oils and additives. Paraffinic oils are preferred. Metalworking straight oils contain

\footnotetext{
${ }^{*}$ Corresponding author.
}

large quantities of additives. Oxidation stability is a very important characteristic for these oils. Also these additives include corrosion inhibitors. The most important additives in metalworking straight oil formulations are the antiwear. Recently, the mixture of anionic/nonionic polymeric surfactants used as additives for metaworking fluid was studied by Omar 2004 [1].

Polyalkylene glycols and esters lubricating oil have a high degree of polarity due to the presence of ether oxygen at every third position of the polymer backbone of glycols and the lone pair of oxygen atom of the ester lin- 
kage. Polyalkylene glycols are often used in combination with extreme pressure additives like fatty acids and phosphate ester [2], where they give synergistic performance. In our previous work, the synthesis of new additives as a pour point and antioxidants was described [3-5]. The author studied new antioxidant for lube oil. This antioxidant dibenzyl s-phenyl thioglyconitrile and other derivatives were prepared by phase transfer catalysts. These compounds were added to oil in different concentrations. A novel method of inhibiting oxidation was proposed by authors. The mechanism was suggested according to surface activity of additive in oil phase. More confirmation for suggested mechanism was investigated by measuring the area occupied per molecule of additive in oil phase. There is a good relationship between the structure of hydrophilic group of the additive and its efficiency. The antioxidants activities of different dosages were evaluated and suggested mechanisms according to micelle and its thermodynamics. The oxidation of the oil has been carried out for different acid formation. Oxidation stability of lube oil was largely affected by sulphur and aromatic hydrocarbons concentration in oil, with the increased sulphur content increasing oxidation stability and time intervals. Polyalkylphenol formaldehyde sulphonate and its ethoxylate were synthesized and evaluated as pour point depressant, viscosity improver and antioxidant. The efficiency of these additives depends on their chemical structure and the degree of mixing mole fraction [3]. The modification of the lyophobic and lyophilic groups, in the structure of the surfactant, may become necessary to maintain surface activity at a suitable level.

Some ester is widely used as lubricants and high performance industrial fluids. They are characterized by good biodegradability, low volatility, good lubricity, good thermal stability and low pour points. Action mechanism of sorbitan palmitate as multifunction additive for pour points depressant, viscosity improver and oxidation of oil was studied elsewhere [5]. The efficiency of this additive depends on its critical micelle concentration. The micelle core acts as a trap for hydrocarbon oxide radicals and the terminal chain of hydrocarbon oxidation. The micellar inhibition depends on incorporation of hydroperoxide or other polar oxygen containing molecules to the reversed micelle, as a result, increasing oxidation stability of oil [5].

Some amphoteric surfactants (N-Decyl-N-benzyl-Nmethylglycine $(\mathrm{AB})$ and N-Dodecyl-N-benzyl-N-methylglycine (CD)) were evaluated by authors. The physico-chemical characteristics were investigated. Surface properties, in particular, the critical micelle concentration (CMC), the maximum surface excess $\left(\Gamma_{\mathrm{CMC}}\right)$ and the minimum surface area $\left(\mathrm{A}_{\mathrm{MIN}}\right)$ were measured. It is found that the surface and thermodynamic properties of the prepared surfactants depend on their hydrocarbon chain length. Also it is found that there is a good relation between surface properties of the additive and their efficiency in depressing the pour point. The mechanism of the depressants action has been suggested according to the adsorption of each additive. Adsorption of the additives on the surface of the wax particles inhibits their growth and alters the crystal habits through micelle core. As a result, the surface and thermodynamic parameters confirm the suggested mechanism and the decreasing of the pour point. This resulted in a multilayer, more isotropic wax crystal, and thus only a fixed amount of wax separates at any given temperatures. The results were discussed in terms of adsorption isotherm [4].

Therefore, it is very important to choose the correct surfactants and optimize its concentration to get full lubricity benefit of using base stock.

The purpose of the present work is to study the prepared poly aromatic ammonium sulphonate (AS) and polyaromatic benzyl triethanolamonium ethoxylate with 6 ethylene oxide units (BS) as additives in metalworking straight oil formulations for antioxidant and antiwear. These additives differ in the head group of surfactant (sulphonate group $\mathrm{SO}_{3}$ and triethanolammonium ethoxylate groups $\left.\mathrm{N}^{+}\left\{\left(\mathrm{C}_{2} \mathrm{H}_{4} \mathrm{OC}_{2} \mathrm{H}_{2}-\mathrm{O}\right)_{6}\right\}_{4} \mathrm{OH}\right)$. The author will suggest the field of the action mechanism of the additive according to micelles inhibition.

\section{Expermental}

\subsection{Synthesis of the Additives}

A sample of light aromatic extract is obtained at refining of lubricting oils. This sample was purified from undesirable components (long chain saturates) and other different impurities e.g. asphaltenes, resins, sulphur compounds and hetrocyclic compounds by active clay. The purified sample was used for preparation different additives.

The ammonium poly aromatic sulphonate was prepared by sulphonation of purified sample of poly aromatic. The prepared polyaromatic was sulphonated with fuming sulphoric acid and neutralized with ammonium solution. The result compound is poly aromatic ammonium sulphonate (AS) which are detected by NMR, and MASS spectroscopy (Figures 1, 2). The purity of the surfactance was about $96.5 \%$ and average molecular weight 444.

The purified sample was chloromethylated in presence of zinc chloride and phase transfere catalyst and purification chloromethylated aromatic and condensed with a stoichiometric amount of triethanol amine according to Omar et al. [6-10]. The product was condensed with ethylene oxide to produce the polyaromatic benzyl trie- 

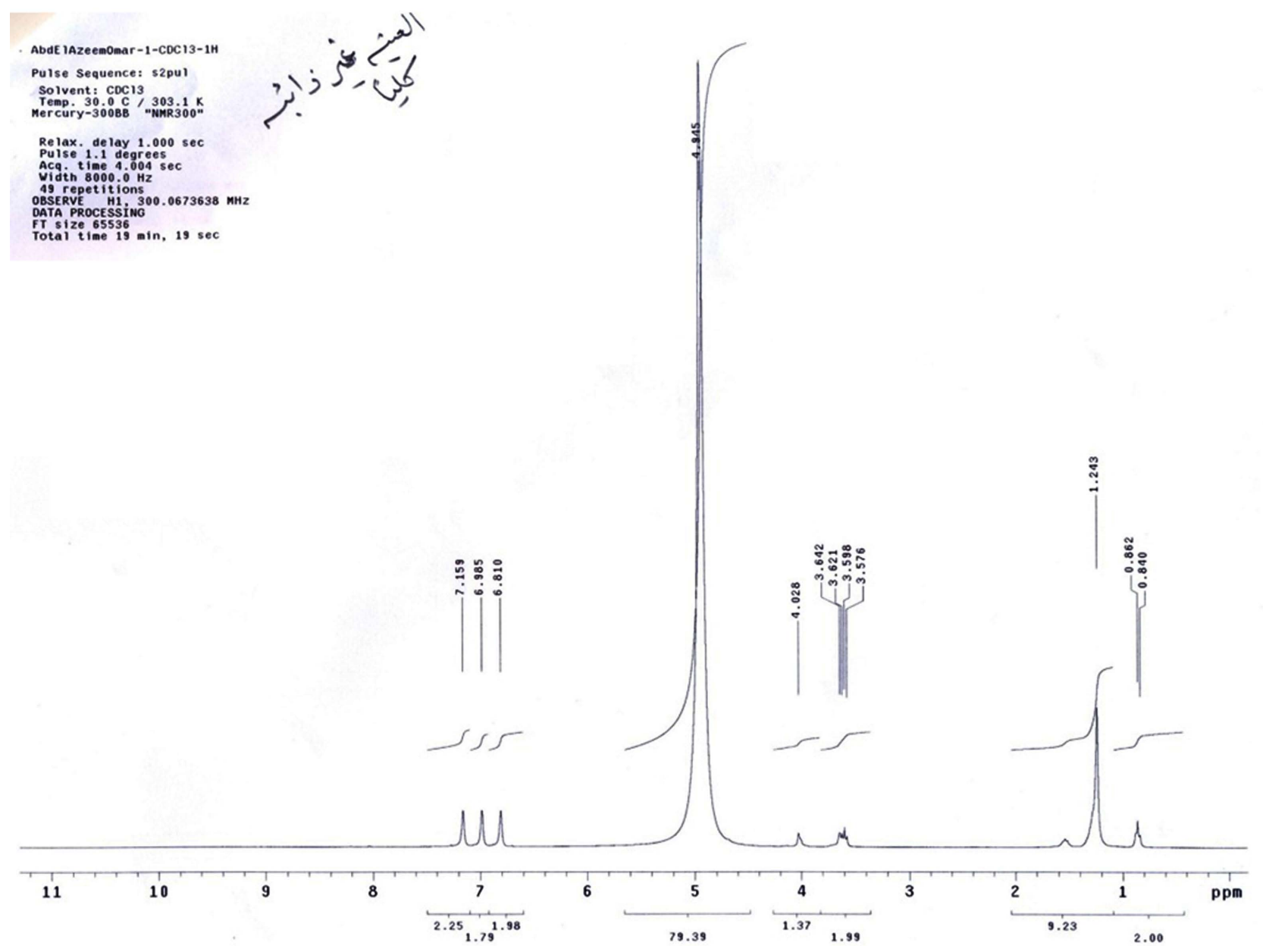

Figure 1. NMR of BS.

thanolamonium ethoxylate with 6 ethylene oxide units (BS). The result compound is poly aromatic ammonium sulphonate (AS) which are detected by NMR, and MASS spectroscopy (Figures 3, 4).

\subsection{Surface and Interfacial Tension Measurements.}

Surface tension of different concentrations for $10^{-7}$ to 0.1 $\mathrm{mol} / \mathrm{L}$ of the synthesized additives was measured by using Kruss Model 8451 in petroleum ether at $30^{\circ} \mathrm{C}$ according to Omar et al. [7].

The physicochemical properties of the base oil are listed in the Table 1 according reverences [3-5].

\section{Formulation of Metal Working Straight Oil}

Base paraffinic oil represent $82 \%$ with additives BS or AS (5\%), other coupling agent about 3\% of dodecyl alcohol and sodium oleate about 7\%.

Wear tests were carried out by Dr. Nora using Pin-onring apparatus under sliding speed of 300, 350, 400 and $450 \mathrm{rpm}$ in wet conditions at room temperaturesin Petro- leum and Petrochemicals Research Institute,KACST $[3,4]$. The samples have the form of cylinderical shape with $8 \mathrm{~mm}$ diameter and $12 \mathrm{~mm}$ long. During the test, the sample was passed against stainless steel ring with normal load of $5 \mathrm{~N}$. Test duration 10 minutes for each sample. Wear of the sample was measured by its weight loss [11]. The best conditions were selected to investigate their worn surface to determine the wear mechanism using scanning electron microscope.

\section{Results and Discussion}

Surface activities of which have been studied from the surface tension versus concentrations curve. It was observed that the critical micelle concentration (CMC) decreases and the surface tension at the CMC increase by the ethoxylation the cationic surfactant. It was found the molecular area occupied at the interface is increased by increasing the number of oxyethylene groups, we choose the best value is 6 ethylen, oxide units. Each ethylene oxide unit is bulkier surface excess concentration $\Gamma$, that of the corresponding nonionic surfactants. This is due to 


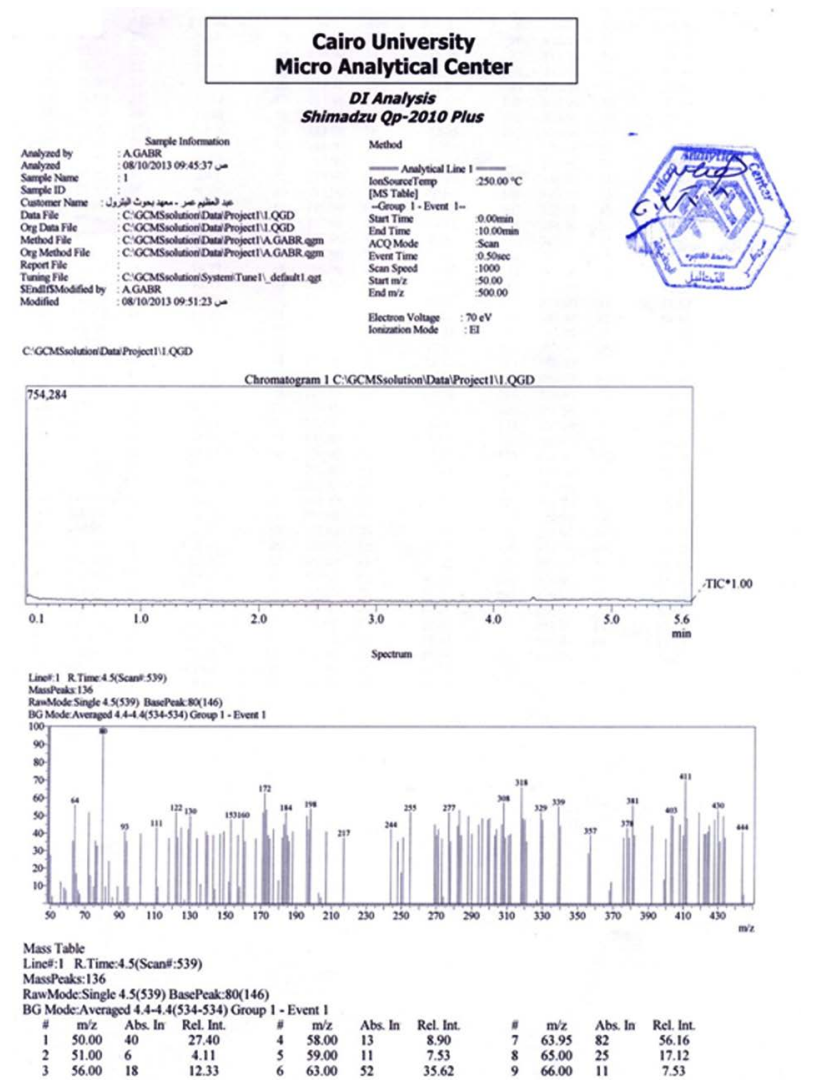

Figure 2. MASS spectroscopy of BS.

AbdE IAZe emOmar-4-CDC13-1H Pulse Sequence: s2pul

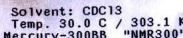

Relax. delay $1.000 \mathrm{sec}$

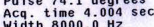

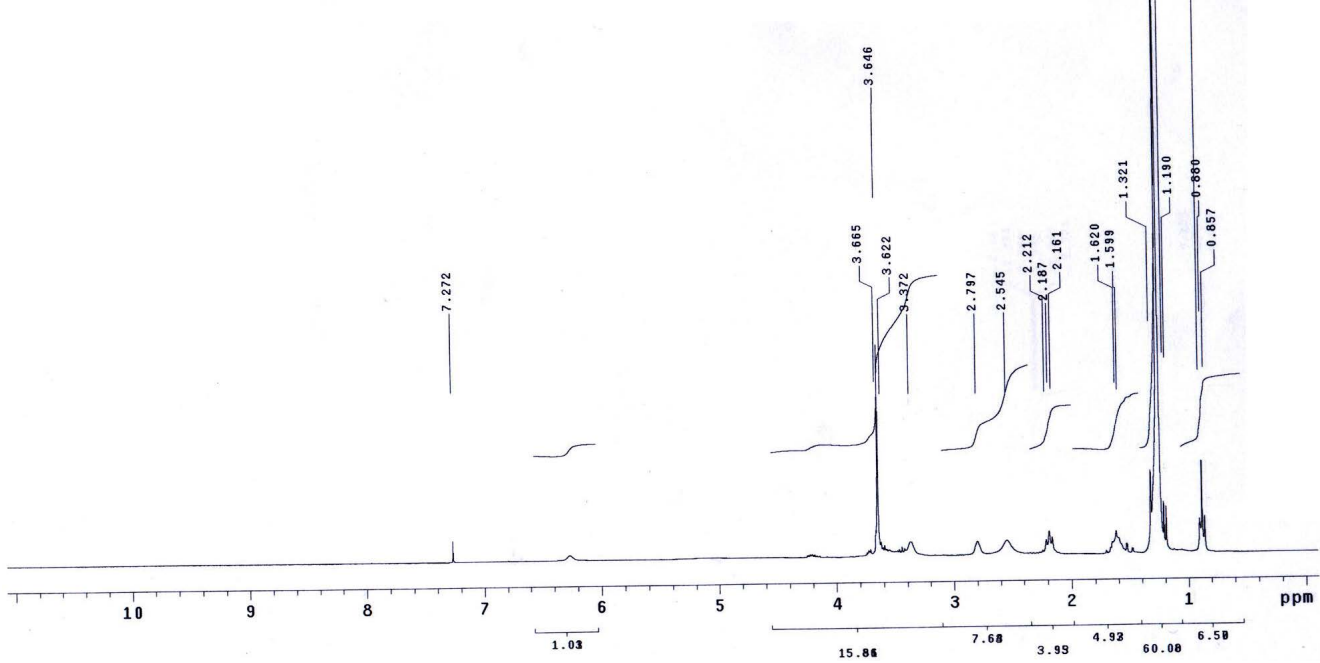

Figure 3. NMR spectrum of AS. 


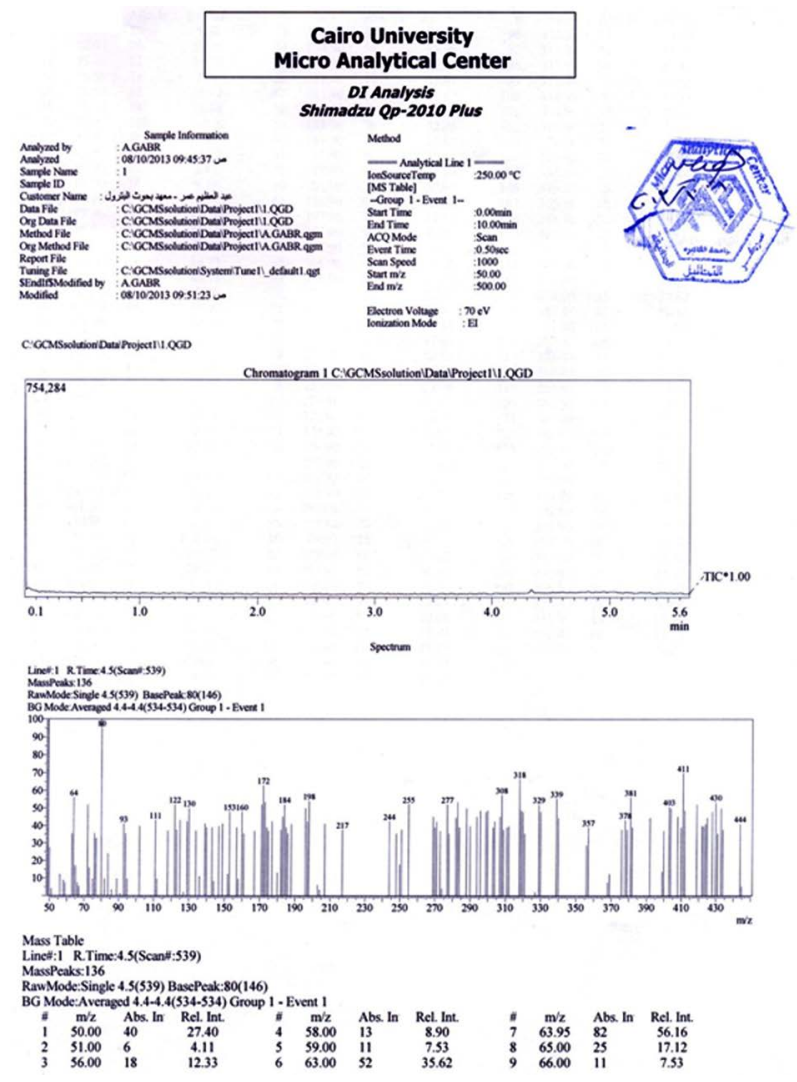

Figure 4. MASS spectrum of AS.

Table 1. The physicochemical properties of the base oil.

\begin{tabular}{lcc}
\hline Properties & Base oil & Test \\
\hline Denisty (g/ml) at 15.5 C & 0.8958 & D. 1298 \\
Refactive index $\mathrm{nD}^{20}$ & 1.4955 & D. 1218 \\
ASTM colour & 4.5 & D. 1500 \\
Kinematic viscoslty cSt & & \\
at 40 C & 17.56 & D. 445 \\
at 100 C & 29.15 & D. 455 \\
Pour point C & 15 & ASTM D 97 \\
Molecular weight & 520 & GPC \\
Total paraffinic content, wr\% & 59.353 & Urea adduction \\
Carbon residue contenty, wt\% & 1.9 & ASTM D524 \\
Ash content, wt\% & 0.0511 & ASTM D482 \\
\hline
\end{tabular}

the electostatic repulsion between the cationic groups. It is observed that the ethoxylated cationic surfactant is more efficient than the corresponding anionic surfactant in lowering the surface tension. The values of surface tension at CMC are close for the anionic surfactant. This is of course, extremely important in utilization of the surfactants (Table 2).

The difference between them is attributed to functional group of each molecule (hydrophilic group). The action of additive of oil phase can be calculated using Gibbs adsorption equation [12]. Comparing the data in Table 3 shows that the CMC value for the additive AS was lower than that of the additive BS, which indicates that the former AS favors micellization processes at a lower concentration than the latter BS. Also, studying the results in Table 3 shows that, the synthesized the additive BS has large values of surface excess and minimum surface area, indicating the BS is the most efficient and gives a greater lowering in surface tension of oil. Thus the change in head group of additive (hydrophilic part) affect of degree of micellization which will be reflect of efficiency of the additive of its activity in oil phase. This concept is agreement with early publication by author [3-5]. These results are compatible with Omar et al. [6-9]. It can be conclude that, the activity of the additive in oil phase enhances by degree of temperature and the values of CMC. This is due to the fact the moiety of molecules increase, as results increase their adsorption rather than giving stable micelle. It has been shown that the changes in structures of hydrophilic and hydrophobic groups strongly affect the shape of the surface tension and therefore, the characteristic properties of the adsorbed film at the oil interface. It has been shown the CMC of this two surfactants non ideal which CMC decreases with in- 
Table 2. Values of surface parameters of synthesized surfactants in oil phase.

\begin{tabular}{lcccc}
\hline Surfactant & $\mathrm{T}\left({ }^{\circ} \mathrm{C}\right)$ & $\mathrm{CMC}(\mathrm{M})$ & $\mathrm{A}_{\mathrm{CMC}}\left(\mathrm{A}^{02)}\right)$ & $\begin{array}{c}\text { Surface tension at } \\
\mathrm{CMC} \mathrm{mN} / \mathrm{m}^{2}\end{array}$ \\
\hline polyaromatic benzyl triethanolamonium & 20 & $9.68 \times 10^{-4}$ & 49 & 11 \\
ethoxylate with 6 ethylene oxide units & 30 & $8.60 \times 10^{-4}$ & 70.9 & 3.45 \\
(BS). & 40 & $8.21 \times 10^{-4}$ & 88.9 & 3.5 \\
poly aromatic ammonium sulphonate & & $8.68 \times 10^{-3}$ & 31.2 & 9.8 \\
(AS) & & $8.50 \times 10^{-3}$ & 29.5 & 13 \\
& & $8.68 \times 10^{-3}$ & 29.1 & 12.7 \\
\hline
\end{tabular}

Table 3. Values of surface parameters of different mole fraction of synthesized surfactants in oil phase.

\begin{tabular}{|c|c|c|c|c|c|}
\hline Mole faction AS/BS & $\mathrm{T}\left({ }^{\circ} \mathrm{C}\right) 30$ & CMC (M) & $A_{\text {CMC }}\left(A^{02)}\right)$ & $\begin{array}{l}\text { Surface tension at CMC } \\
\mathrm{mN} / \mathrm{m}^{2}\end{array}$ & $\Gamma_{\text {CMC }}\left(10^{-10}\right)$ \\
\hline 0.2 & & $7.68 \times 10^{-4}$ & 79 & 10 & 4.45 \\
\hline 0.4 & & $2.60 \times 10^{-4}$ & 94.9 & 10 & 5.5 \\
\hline 0.6 & & $1.21 \times 10^{-5}$ & 116.9 & 11 & 3.89 \\
\hline 0.8 & & $2.5 \times 10^{-5}$ & 98.7 & 12.5 & 2.5 \\
\hline
\end{tabular}

creasing mole fraction of AS/BS (Table 3). Science there are no difference between hydrophobic tail of the two surfactants, the area per molecule of the mixed micelle is independent of the micelle composition, which calculated according to Omar [12]. The deviation from ideal mixing is rather small, because of the small magnitude of the strike repulsion. Consequently, these surfactants mixtures can behave partly as ionic single chain molecules and partly as zwitterionic paired chain molecules. We can conclude that, the incorporation of oxyethylene groups between the hydrocarbon chain and the hydrophilic group changes the properties of the cationic surfactant and the anionic surfactant. The CMC decreases and the minimum area at CMC increases by the ethoxylation. It were found that the molecular area occupied at the interface is increased by increasing the ratio of AS surfactant. This is clearly due to the electrostatic repulsion between cationic groups.

\section{Antiwear Properties}

The effectiveness of tested surfactants is very good for two additives and additive.

Concentrations used and depend on their critical micelles. The CMC concentrations gave significant wear reduction. The additive forms a film between the metal surface and the rotating ring that minimize the wear rate. Figure 5 shows polyaromatic benzyl triethanolamonium ethoxylate with 6 ethylene oxide units (BS) is the best antiwear additive among the other poly aromatic ammonium sulphonate (AS) additive. With increasing the sliding speed to $600 \mathrm{rpm}$, the worn surface seems rougher compared to other worn at $300 \mathrm{rpm}$, rpm, the worn surface was totally damage as shown in Figure 5. On the other hand addition different concentration of each addi- tive at constant speed $400 \mathrm{rpm}$ lead to increase wear resistance and the efficiency of BS additive is preferable than additive AS (Figure 6). This mean that the efficiency of reduction wear at $500 \mathrm{rpm}$ as the following, $48.98 \%$ and $23.471 \%$ for additives BS and AS respectively (Figure 7). This behavior is due to the dependence of inhibition efficiency on the $A_{\min }$ and the degree of surface coverage area of the additive (Figures 7,8 ).

The investigation of the ability of additives and their mixtures as anti wear are shown in Figure 9. It is clear that, wear resistance are improved by increasing the additive concentrations. The optimum value appear at the critical micelle concentration of additive and at mole fraction of AS/BS equal 0.6. These results can be discussed according the surface activity of the additive like $\mathrm{A}_{\min }$, critical surface tension at CMC and surface excess concentration $\Gamma_{\mathrm{CMC}}$ (Tables 2, 3). The additions of AS to BS form mixture exhibiting pettier performance in reduction wear (Figure 9). The author confirms degree of mixing has predominant factor in enhance property of oil. This is due to stability of micelle increase with increasing ratio of AS and BS decrease until mole fraction equal 0.6 of AS/BS. As the results increase are occupied per molecule, consequently improve wear resistance of oil. Moreover, these results are more confirmed by increasing the activity of each additive in oil phase which has surface property depend on CMC and degree of mixing (Table 3).

The effect of these additives on the oxidation stability of oil is given in Figure 10. The data show the additive retards the oxidation of oil for limit time and loss its efficiency after 50 hours. Comparing between two additives in increasing oil stability, the additive BS is the best; due to it has the best surface properties. The mole fraction 0.6 of BS/AS is the best in its oxidation stability. The author 


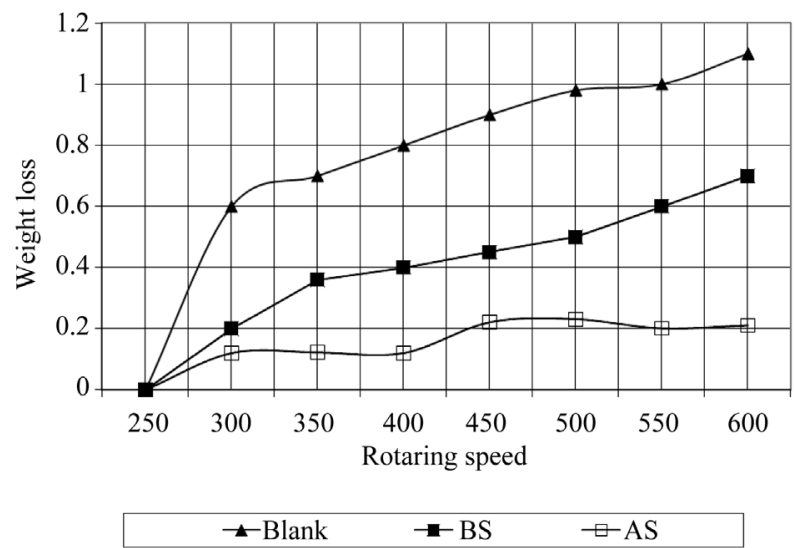

Figure 5. Effect of rotaring speed and additives on wearing using lube oil.

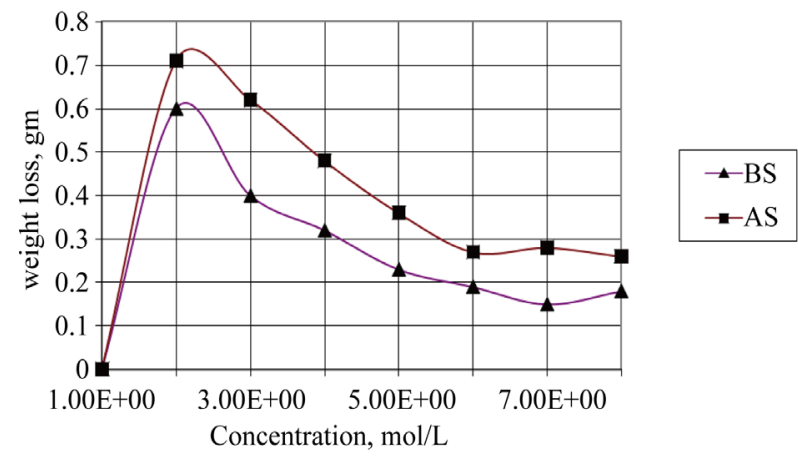

Figure 6. Effect of the additive concentration on wear resistance at speed $400 \mathrm{rpm}$ at constant conditions.

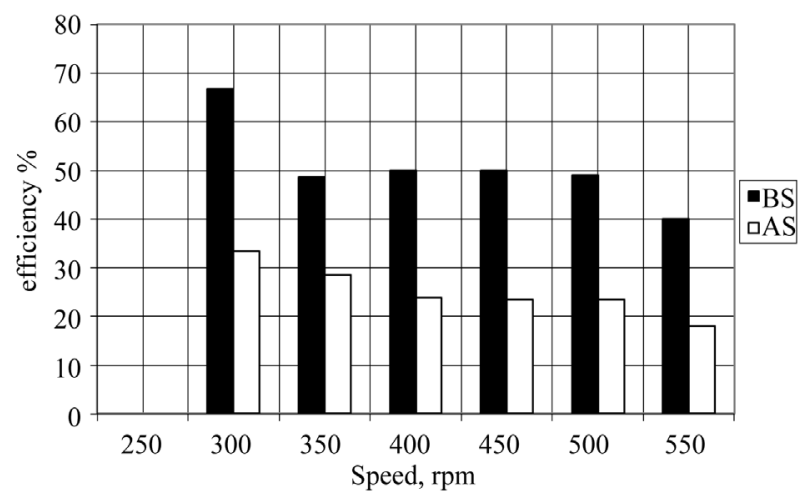

Figure 7. Effect of the two additives on wear resistance at speed.

concludes that the ability and stability of micelle is predominant factor for increase oxidation stability of oil. The micelle and inhibit propagation of free radicals and terminate reaction processes of free radicals as discussed early $[4,5]$. More confirmation, the increasing surface area occupied per molecule of mixture attend at mole fraction 0.6 BS/AS show surface properties of iron samples by using additives BS and AS at optimum conditions.

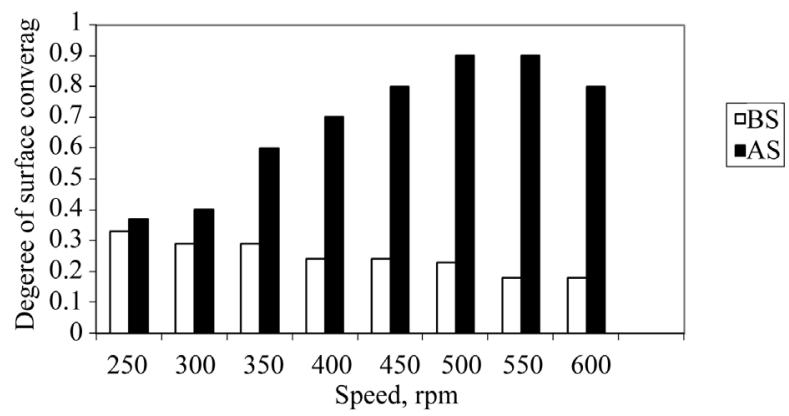

Figure 8. Effect of the degree of surface coverage of the additive on wear resistance.

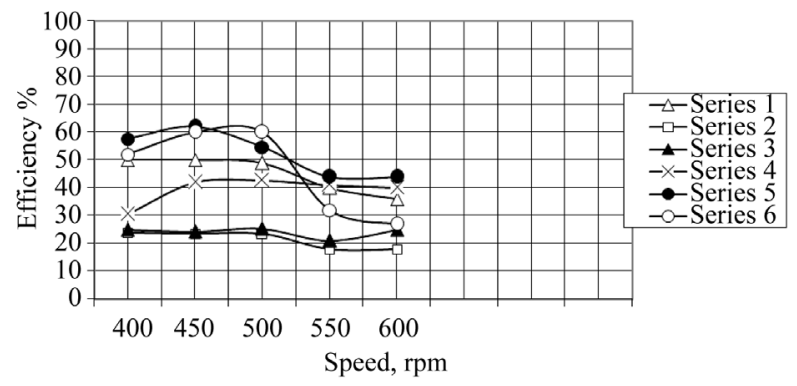

Figure 9. Effect of the different mole fraction of AS/BS on wear resistance at different speed rpm.
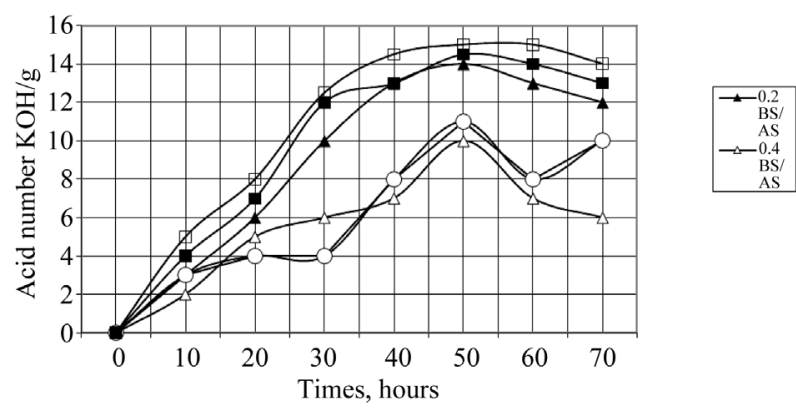

Figure 10. Effect of the different AS and BS and their meture on acid number $\mathrm{KOH} / \mathrm{gm}$.

It is clear that additive BS adsorbed on the metal surface forming thin film and prevent petting, while additive less efficient and pitting occur in the metal surface (Figure 11).

\section{Conclusions}

The results strongly indicate that the antiwear and antioxidants effectiveness of all additives (AS, BS) depends on chemical composition, concentration and sliding speed.

The oxidation stability of oil as measured by total acid number indicates that, the oxidation inhibitor efficiency follows the order.

BS/AS > BS $>$ AS and the mole fraction affect on the degree of oil stability. These results depend on the value 

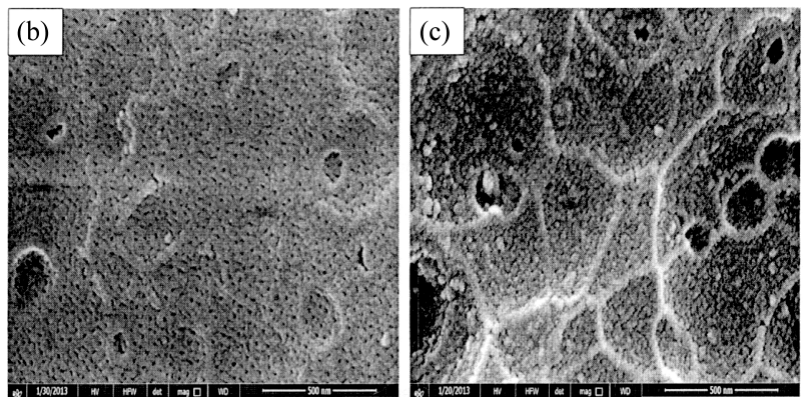

Figure 11. EDX of the surface of metal for using BS (b) and ES (c) at optimum conditions.

of CMC and area occupied per molecule in oil phase.

Further work will study the above additives as corrosion inhibitors and discuss the results according to the composition of mixed micelles and measure the hydrophilic and hydrophobic balance of additives HLB.

\section{REFERENCES}

[1] A. M. A. Omar, "Micellization and Adsorption of Anionic/ Nonionic Polymeric Surfactants for Metal Working Fluid at Different Interfaces,” Industrial Lubrication and Tribology, Vol. 56, No. 3, 2004, pp. 171-176.

[2] I. Minami, S. Kikuta and H. Okabe, "Anti-Wear and Friction Reducing Additives Composed of Orth-Phenylene Phosphate-Amine Salts for Polyether Type Base Stocks, Tribology International, Vol. 31, No. 6, 1998, pp. 305312. http://dx.doi.org/10.1016/S0301-679X(98)00038-3

[3] N. Elmehbad, "Development Antioxidants Synthesized by Phase Transfer Catalysts for Lubricating Oil,” Biotech Conference \& Expo, 12-16 May 2013.
[4] N. Elmehbad, "Developments of Multifunctional Additives for High Quality Lube Oil,” Journal of Power and Energy Engineering, Vol. 1, No. 5, 2013, pp. 84-89.

[5] N. Elmehbad, "The Development and Application of Ester for Lubricating Oil by Phase Transfer Catalysts,” Accepted for Oral Presentation in 19th International Colloquium, 21-23 January 2013, Germany.

[6] T. T. Khidr, E. M. S. Azzam, Sahar, S. Mutwaa and A. M. A. Omar, "Study of Some Anionic Surfactants as Pour Point Depressant Additives for Wax Gas Oil,” Industrial Lubrication and Tribology, Vol. 59, No. 2, 2007, pp. 6468. http://dx.doi.org/10.1108/00368790710731855

[7] T. T. Khidr and A. M. A. Omar, "Anionic/Nonionic Mixture of Surfactants for Pour Point Depression of Gas Oil,” Egyptian Journal of Petroleum, Vol. 12, 2003, pp. 21-26.

[8] T. T. Khidr, D. Ismail and A. M. A. Omar, "Improving the Flow Properties of n-Paraffin Gas Oil by Cationic and Non Ionic Surfactants," Journal of Faculty of Education, Vol. 25, 2000, pp. 121-135.

[9] A. M. A. Omar, "Separation of Emulsifiable Oil from Solution by Surface Tension Control," Adsorption Science and Technology, Vol. 19, No. 1, 2001, pp. 91-100. http://dx.doi.org/10.1260/0263617011494006

[10] A. M. A. Omar, "Correlation between Surface, Thermodynamic Parameters of the Anionic Collector and Oil Separation by Flotation," Journal of Petroleum Science and Technology, Vol. 19, No, 7-8, 2001, pp. 911-921.

[11] A. El Sayed el Tabei, "Production of Surfactants from Petroleum by Products by Phase Transfer Catalysis for Metal Working Fluids," Ph.D. Thesis, Ain Shams University, Cairo, 2008.

[12] A. M. A. Omar, “Anionic/Nonionic Collector Efficiency as Related to the CMC of the Mixture," Afinidad, Vol. 464, 1996, pp. 262-266. 